\title{
Low-energy CZT detector array for the ASIM mission
}

Cenkeramaddi, Linga Reddy; Genov, Georgi; Kohfeldt, Anja; Njoten, Kare; Rostad, Maja Elise; Skogseide, Yngve; Roscoe, Magnus; Solberg, Arne; Stadsnes, Johan; Ullaland, Kjetil

Total number of authors:

15

Published in:

Proceedings of IEEE Instrumentation/Measurement Technology Conference (I2MTC)

Link to article, DOI:

10.1109/I2MTC.2012.6229184

Publication date:

2012

Document Version

Publisher's PDF, also known as Version of record

Link back to DTU Orbit

Citation (APA):

Cenkeramaddi, L. R., Genov, G., Kohfeldt, A., Njoten, K., Rostad, M. E., Skogseide, Y., Roscoe, M., Solberg, A., Stadsnes, J., Ullaland, K., Østgaard, N., Budtz-Jørgensen, C., Kuvvetli, I., Mikkelsen, S., \& Mæhlum, G. (2012). Low-energy CZT detector array for the ASIM mission. In Proceedings of IEEE Instrumentation/Measurement Technology Conference (I2MTC) (pp. 2119-2123). IEEE. https://doi.org/10.1109/I2MTC.2012.6229184

\section{General rights}

Copyright and moral rights for the publications made accessible in the public portal are retained by the authors and/or other copyright owners and it is a condition of accessing publications that users recognise and abide by the legal requirements associated with these rights.

- Users may download and print one copy of any publication from the public portal for the purpose of private study or research.

- You may not further distribute the material or use it for any profit-making activity or commercial gain

- You may freely distribute the URL identifying the publication in the public portal 


\section{Low-energy CZT detector array for the ASIM mission}

Linga Reddy Cenkeramaddi, Georgi Genov, Anja Kohfeldt, Kåre Njøten, Maja Elise Rostad, Yngve Skogseide, Magnus Roscoe, Arne Solberg, Johan Stadsnes, Kjetil Ullaland, Nikolai Østgaard University of Bergen

Department of Physics and Technology Bergen, Norway

\author{
Carl Budtz-Jørgensen, Irfan Kuvvetli \\ Technical University of Denmark \\ National Space Institute \\ Copenhagen, Denmark \\ Sindre Mikkelsen, Gunnar Mæhlum \\ Gamma Medica-Ideas (Norway) as \\ Oslo, Norway
}

\begin{abstract}
In this article we introduce the low-energy CZT (CdZnTe) 16 384-pixel detector array on-board the Atmosphere Space Interaction Monitor (ASIM), funded by the European Space Agency. This detector is a part of the larger Modular Xand Gamma-ray sensor (MXGS). The CZT detector array is sensitive to photons with energies between $15 \mathrm{keV}$ and $400 \mathrm{keV}$. The principal objective of the MXGS instrument is to detect Terrestrial Gamma ray Flashes (TGFs), which are related to thunderstorm activity. The concept of the detector array is presented, together with brief descriptions of its mechanical structure and electronics. A method for detector characterization is outlined and preliminary experimental results are shown.
\end{abstract}

Index terms: Gamma ray detector, X-ray detector, Radiation Imaging, CZT detector, International Space Station, MXGS instrument, ASIM mission, Terrestrial Gamma Flashes.

\section{INTRODUCTION}

The Atmosphere-Space Interaction Monitor (ASIM) payload is scheduled to be flown on the Columbus External Platform Facility (CEPF) of the International Space Station (ISS), with a planned launch in 2014. The main objective of ASIM is to observe Terrestrial Gamma Flashes (TGFs) and Transient Luminous Events (TLEs), known as blue jets, sprites and elves and how these phenomena are related to thunderstorms and lightning discharges. TGFs were discovered in 1994 as short flashes $(<1 \mathrm{~ms})$ of high energy photons [1]. Photons with energies up to $40 \mathrm{MeV}$ have been reported recently [2], which make the TGF the most energetic natural photon phenomenon that exists on Earth. Although we know that TGFs are produced below $20 \mathrm{~km}$ altitudes [3],[4] and related to the lightning discharges, there are many open questions related to their production mechanism and their importance and impact on the Earth's electric circuit. ASIM is designed to address these open questions.

ASIM payload consists of two main scientific instruments [5]-[9]. The first one is the Modular Multispectral Imaging Array (MMIA) composed of two cameras and two photometers. The second one is MXGS, composed of two layers, the pixelated ( $>16000$ pixels) $\mathrm{CZT}$ array, $1024 \mathrm{~cm}^{2}$ in area and $0.5 \mathrm{~cm}$ thick and a Bismuth Germanate (BGO) layer, $900 \mathrm{~cm}^{2}$ in area and $3.2 \mathrm{~cm}$ thick. The CZT (semiconductor) layer will detect photons in the energy range $15-400 \mathrm{keV}$, while the BGO (scintillator) layer can detect photons up to $20 \mathrm{MeV}$. A coded mask that will stop about $50 \%$ of the photons below $200 \mathrm{keV}$ will allow for imaging the low energy photons. Hence, MXGS will be the first instrument to routinely image TGFs and will have sensitivity 10 times what has been flown to date. The ASIM payload is designed and built by a collaboration between the Danish Technological University (DTU), University of Bergen (UB), University of Valencia (UV) and Polish Academy of Science (PAS).

The focus of this paper is the Cadmium Zinc Telluride (CZT) detector array of the MXGS instrument.

\section{CZT LOW-ENERGY DETECTOR ARRAY - DESIGN CONCEPTS}

\section{A. Design overview}

The complete CZT detector module consists of the detector array built from $64 \mathrm{DMs}, 256$ pixels each, made from 4 Detector Assembly Units (DAUs). The 4 DAUs are mounted on the Detector Assembly Frame. Each DAU contains the necessary read-out electronics, $16 \mathrm{DMs}$ and a combined thermal and mechanical structure. Thus, one DAU has 4096 pixels and the entire CZT detector array features 16384 pixels. Each of these pixels detects energy spectra of the incident photons up to $400 \mathrm{keV}$. An exploded view of a DAU, together with a schematic of the complete detector array is shown in Fig. 1.

The read-out electronics design contains a Field Programmable Gate Array (FPGA), analog read-out electronics and connectors for the external interfaces to the DPU and will host 16 detector modules. The FPGA contains the Read-out Control Unit (RCU) and glue logic towards the read-out electronics, for example the ADCs. Each detector module is built from one ASIC module [10] which contains two XA ASICs from Gamma Medica-Ideas (Norway) and one CZT module. The XA ASICs are designed to be connected to a multi-drop current mode bus for energy, trigger and pixel 
address read-out. We have chosen to use 4 read-out chains with 4 detector modules each. The number of parallel DMs is a trade-off between circuit complexity, number of external components, power consumption and maximum obtainable event-rate. The chosen chain size of 4 DMs is capable of handling the required burst-rate, whilst keeping the number of external components at a reasonable level. The performance objectives for the CZT detector layer are given in Table I.

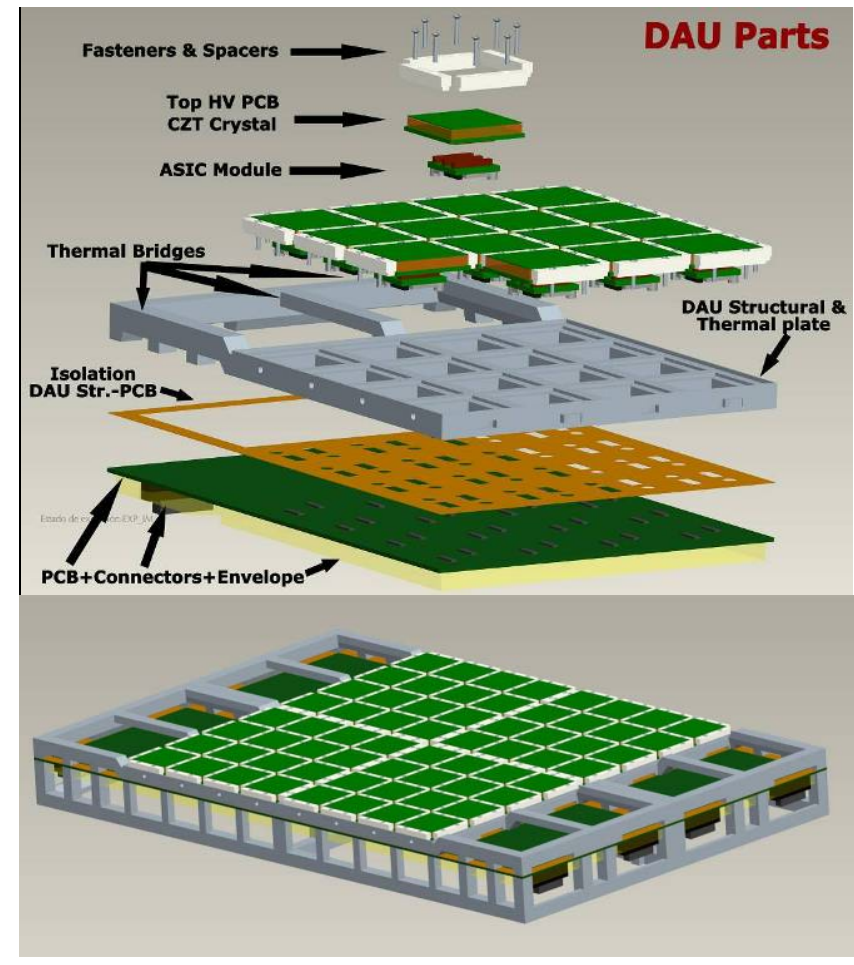

Figure 1. Schematics of one DAU (top) and of the complete CZT detector array (bottom).

TABLE I. SPECIFICATION OF PERFORMANCE PARAMETERS FOR THE CZT DETECTOR LAYER

\begin{tabular}{|c|c|}
\hline Description & Value \\
\hline Energy range & $15 \mathrm{keV}$ to $400 \mathrm{keV}$ \\
\hline Energy resolution & $<10 \%$ at $60 \mathrm{keV}$ for $95 \%$ of the pixels \\
\hline Total efficiency per CZT module & $>94 \%$ at $100 \mathrm{keV}$ \\
\hline Relative time accuracy & Better than $10 \mu \mathrm{s}$ \\
\hline Burst rate capability & $350 \mathrm{cts} / \mathrm{ms}$ \\
\hline
\end{tabular}

\section{B. The detector module}

The detector module contains a CZT module and an ASIC module.

The CZT module consists of four single-crystal CZT submodules, bonded on one Arlon substrate (PCB). Each CZT sub-module is divided into 64 pixels ( $2.5 \mathrm{~mm}$ pixel pitch), thus yielding a total of 256 pixels per DM. On top of the CZTs there is a PCB with conductive hatched grid pattern, facing down towards the CZT, for HV distribution to all pixels. On the top, facing upwards, there are eight small solder points for connection to the High Voltage Supply. Three connectors are mounted on the bottom side of the PCB (CZT substrate) for connection to the ASIC module.

Each ASIC module includes two XA-ASIC chips, and each ASIC chip has 128 channels. The ASICs are mounted on the bottom side of a PCB covered by a heat sink. Three connectors are mounted on the top of the PCB, for connection to the CZT module. The ASIC module connected to the CZT module forms the Detector module (DM). The two connectors on the ASIC bottom side connect the DM module to the DAB.

\section{Readout Electronics}

The purpose of the readout electronics is to provide an interface between the detector modules and the Data Processing Unit (DPU), as illustrated in Fig. 2. It includes circuitry to digitize the analog outputs, including pixel address and multi-hit outputs, from the XA ASICs; circuitry to configure the XA ASICs; implementation of a suitable communications link between the DAU RCU and the DPU. The DAU RCU is controlled by an Actel RTAX2000SCGA624 FPGA without an embedded Central Processing Unit (CPU), i.e. a pure firmware solution. The FPGA is clocked with an external crystal at $36 \mathrm{MHz}$. It monitors the detector module for events; controls the ADCs; provides an internal $1 \mathrm{MHz}$ clock for time tagging of events; configures the XA ASICs via serial links; supports memory read and write commands to provide status information to the DPU; provides an event FIFO (First-In-First-Out) for buffering in case the event rate exceeds the link capacity; offers real-time spectral binning.

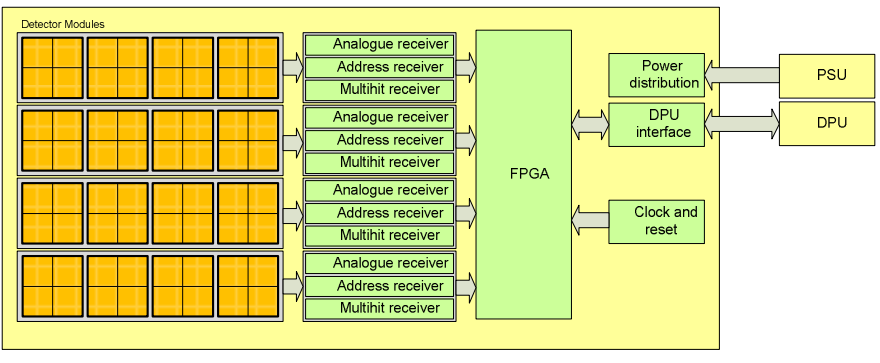

Figure 2. CZT-DAU overview. The $4 \times 4$ detector modules are shown in orange. The analogue read-out electronics is organized in 4 separate chains which are interfaced digitally to the FPGA.

PSU - Power Supply Unit. DPU - Data Processing Unit.

The XA-ASICs is self-triggered and data-driven ASIC, delivering energy and position information of up to 128 channels. It is normally used in medical imaging applications. Getting pulses from the CZT module, the ASIC shapes, stretches and amplifies the analog signal. If this signal exceeds a given threshold, a trigger signal is generated together with an address signal and the analog energy signal. The amplitude of the final trigger signal is proportional to the number of hits per time slot so that multi-hits are recognizable. In order to get the best performance, there are configuration possibilities for both the whole chip and individual channels. To configure the chip, the XA ASIC 1.82 has a configuration register. Since the configuration register is volatile, the register file must be downloaded onto the ASIC after power up. 
Further analog functions are implemented for the treatment of the signals after they have left the ASIC. Those include reception of pixel and ASIC address, energy output (the output representing the energy of any detected Gamma-ray photon hit) and trigger output, indicating that one or more pixels have been struck with a photon (multi-hit).

\section{DETECTOR MODULE CHARACTERIZATION AND CONCLUSIONS}

CZT detectors have been characterized in detail previously [11]. There, the authors investigated the detector pixel by pixel by scanning it with a narrow beam. This procedure is accurate but time-consuming, and the setup is too complex for routine calibrations and diagnostic work while in space.

\section{A. Outlier pixels}

We have performed tests, using ${ }^{241} \mathrm{Am}(60 \mathrm{keV})$ as a source. Our observations confirm the conclusion that there is a variation in the efficiency from pixel to pixel, resulting in different count rates. However, one may observe outlier pixels in which the total number of counts is either by an order (or orders) of magnitude larger or containing just a couple of pulses (see Fig. 3). Such pixels are incapable of registering spectral data and should be switched off.
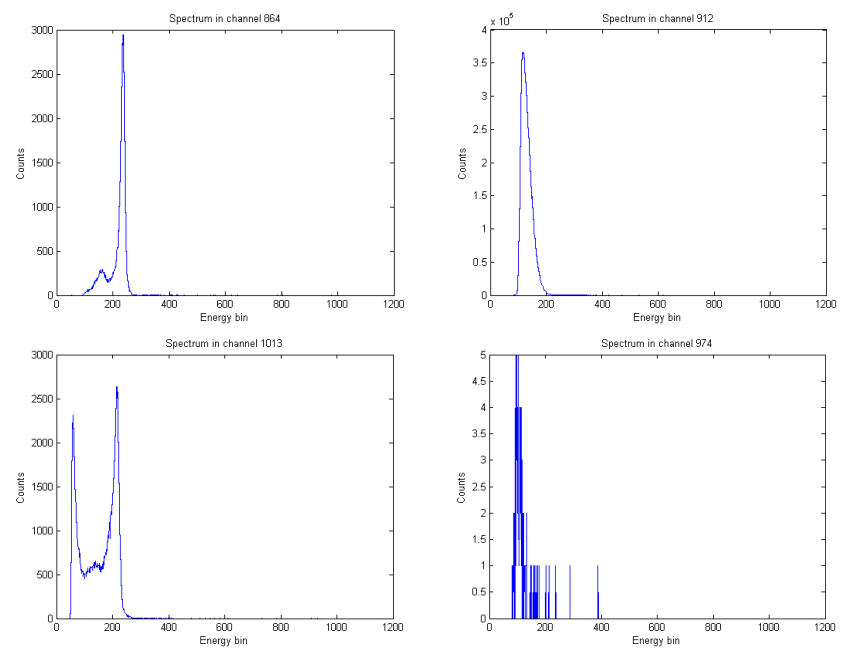

Figure 3. Spectra in pixels illuminated with ${ }^{241} \mathrm{Am}$. In the top-left graph is shown a pixel with a good ${ }^{241} \mathrm{Am}$ spectrum; the pixel in the top-right plot is noisy and incapable of registering spectral data. The pixel in the bottomright registers only a couple of counts and is also incapable of registering spectral data. The pixels from the right column should be switched off. The graph from bottom-left shows a noisy pixel, which can be used for energies somewhat higher than $15 \mathrm{keV}$. In this latter case, one may consider adjusting the threshold of this pixel. This is, however, equivalent to switching off the noisy energy bins on the left.

The procedure used to automatically detect candidates for switch-off is as follows: the spectrum in every channel/pixel is integrated, thus yielding the total counts in every pixel. This is done per-ASIC, i.e. in batches of 128 pixels. A histogram of the integrals within a batch/ASIC is built (Fig. 4). The mean, $\bar{X}$ (1) and the standard deviation, $\sigma$ (2), of the distribution of the integrals are determined, using Student's tdistribution:

$$
\begin{gathered}
\bar{X}=\frac{1}{n} \sum_{i=1}^{n} X_{i} \\
\sigma^{2}=\frac{1}{n} \sum_{i=1}^{n}\left(X_{i}-\bar{X}\right)^{2}
\end{gathered}
$$

As an outlier is defined every pixel whose total integral is larger or smaller than $2 \sigma$. The spectra in all those pixels are considered dead in the following stage of estimating the parameters of the spectra.

\section{B. Preliminary estimation of spectrum parameters}

The estimated parameters per pixel are peak position, Full Width at Half Maximum (FWHM), energy resolution and peak integral. No information on efficiency is generated at this stage because no reference is provided and the analysed pixels are those that have NOT been excluded as dead in the previous round.

\section{- Peak position}

Our peak detection algorithm detects peaks that have a height bigger than a certain threshold. Due to noise at the top of the peak in some pixels, the algorithm may detect the same peak twice. To overcome this, a minimum distance between two peaks is predefined. Any two peaks situated within this minimum distance are considered as one. This is justified since our calibration source has only one gamma-line.

- FWHM and energy resolution

Once a peak is detected, its FWHM, defined as QTY (quantity) channels is found. This is done geometrically by dividing the peak height in two and finding the number of channels the peak occupies.

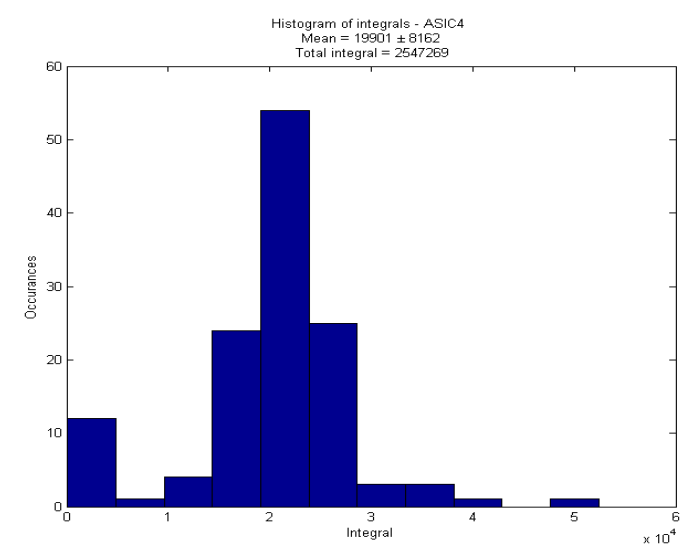

Figure 4. Distribution of the total counts per pixel. All pixels with spectrum integral beyond $2 \sigma$ are to be turned off.

The algorithm finds the aforementioned number of channels only on the right-hand-side of the peak maximum and multiplies it by two in order to get the FWHM (QTY channels). This, in addition to the fact that no background correction under the peak is done, introduces a certain asymmetry in the results per pixel, which is presently 
considered small enough to be neglected in the overall context of one DAU.

Using the peak position and the knowledge of the energy in the gamma-line, the width of the energy bin (channel) is defined. Note that as a second calibration point the origin of the coordinate system is automatically assumed. This is not a perfect energy calibration, but it is accepted here as a good first order approximation. On this basis the value of the FWHM in energy units is deduced.

The energy resolution is then found in the classical way by dividing the FWHM by the energy of the gamma-line. Preliminary results are shown in Fig. 5. The results show resolution better than $10 \%$ in more than $95 \%$ of the pixels. This is well within the requirements for this instrument.
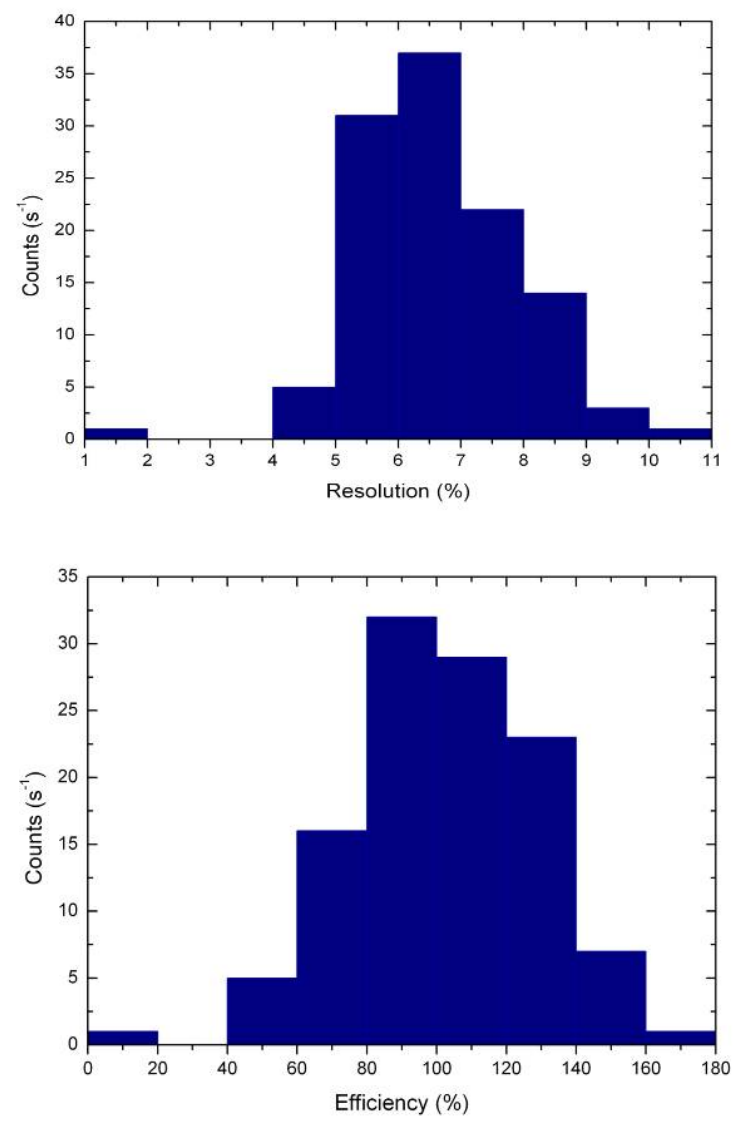

Figure 5. Top - distribution of the 128 pixels in the ASIC from Figure 4 according to their resolution. Bottom - the efficiency distribution of the same pixels. The pixels with resolution between $1 \%$ and $2 \%$ and with efficiency between $0 \%$ and $20 \%$ are false hits, i.e. peaks incorrectly detected by the algorithm.

- Peak integral

The peak integral is not calculated by numerically integrating the peak, since that would require finding the peak borders. To evade this problem, the peak integral is computed from the FWHM (QTY channels) and the peak height. This is done using the Gaussian distribution with an area $A(3)$ :

$$
f(x)=\frac{A}{\sigma \sqrt{2 \pi}} \exp \left(-\frac{X-\bar{X}}{2 \sigma^{2}}\right)
$$

The maximum peak height, $h_{\max }(4)$, is found at $X=\bar{X}$ and

$$
h_{\max }=\frac{A}{\sigma \sqrt{2 \pi}}
$$

Given that

$$
F W H M=2 \sigma \sqrt{2 \ln (2)}
$$

It is found that

$$
A=\frac{h_{\mathrm{max}} F W H M}{2 \sqrt{2 \ln (2)} \sqrt{2 \pi}}=0.937 h_{\max } F W H M
$$

The value obtained this way is divided by a reference value and this yields the relative efficiency. The selected reference value here is the mean of the efficiency within the selected ASIC and, therefore, the centre of the distribution is at $100 \%$. The results obtained this way are not yet directly comparable with the system requirements. Further calculations will be performed in the future to obtain such comparable data.

\section{ACKNOWLEDGEMENTS}

This project is supported by European Space Agency as a subsub-contract between DTU and UB, to the contract 4000101107/10/NL/BJ between ESTEC and TERMA Ref. and by ESA PRODEX under contract C4000102100 and by Norwegian Research Council under contract 184790/V30.

\section{REFERENCES}

[1] G. J. Fishman, et al., "Discovery of intense gamma-ray flashes of atmospheric origin," Science, 264(5163), pp. 1313-1316, 1994.

[2] M. Marisaldi, et al., "Detection of Terrestrial Gamma-Ray Flashes up to $40 \mathrm{MeV}$ by the AGILE satellite," J. Geophys. Res, 115, A00E13, 2010.

[3] T. Gjesteland, N. Østgaard, P. H. Connell, J. Stadsnes, and G. J. Fishman, "Effects of deadtime losses on Terrestrial Gamma ray Flash measurements done by the Burst And Transient Source Experiment," J. Geophys. Res., 115, A00E21, 2010.

[4] B. E. Carlson, N. G. Lehtinen and U. S. Inan, "Constraints on terrestrial gamma ray flash production from satellite observation," Geophys. Res. Lett., 34, L08, 809, 2007.

[5] ASIM Topical Team meeting, http://www.dsri.dk/atmosphere/asim/tt1/tt1.html (last accessed, Nov. 2011).

[6] P. L. Thomsen, "ASIM Payload System Overview," ASIM Topical Team Meeting-1, ESA/ESTEC, June 26-27, 2006.

[7] T. Neubert, et al., "The Atmosphere-Space Interactions Monitor (ASIM) for the International Space Station," ILWS (International Living With a Star) Workshop 2006, Goa, India, Feb. 19-20, 2006.

[8] G. Reibaldi, et al., "The ESA Payloads for Columbus- A bridge between the ISS and exploration," ESA Bulletin, No 122, May 2005, pp. 60-70. 
[9] T. Neubert, et al., "The Atmosphere-Space Interactions Monitor (ASIM) for the International Space Station," Workshop on Coupling of Thunderstorms and Lightning Discharges to Near-Earth Space, June 2327, 2008, University of Corsica, Corte, France.

[10] S. Mikkelsen, et al. "A Low Power and Low Noise Multi-Channel ASIC For X-Ray and Gamma-Ray Spectroscopy in Space," Second
International Workshop on Analog and Mixed Signal Integrated Circuits for Space Applications (AMICSA 2008), 31 August - 2 September 2008.

[11] C. Budtz-Jørgensen, I Kuvvetli, Y. Skogseide, K. Ullaland and N. Østgaard, "Characterization of CZT detectors for the ASIM mission," IEEE Transactions on Nuclear Science, vol. 56, 4, 2009, pp. 1842-1847. 